\title{
Body weight is a robust predictor of clinical progression in
}

\section{Huntington disease}

Running head: Weight loss and disease progression in HD

\section{Authors \& affiliations:}

Jorien M.M. van der Burg, MD, PhD ${ }^{1}$; Sarah L. Gardiner, MD²; Albert C. Ludolph, MD, PhD ${ }^{4}$; G. Bernhard Landwehrmeyer, MD, PhD"; Raymund A.C. Roos, MD, PhD²; N. Ahmad Aziz, MD, PhD²,3,5

Departments of ${ }^{1}$ Public Health and Primary Care, ${ }^{2}$ Neurology and ${ }^{3}$ Epidemiology, Leiden University

Medical Centre, Leiden, the Netherlands

${ }^{4}$ Department of Neurology, Ulm University Hospital, Ulm, Germany

${ }^{5}$ Department of Neurodegenerative Disease, UCL Huntington's Disease Centre, University College London Institute of Neurology, London, United Kingdom

Corresponding author:

Dr. N.A. Aziz. Department of Neurology, Leiden University Medical Centre, Leiden, the Netherlands \& Department of Neurodegenerative Disease, UCL Huntington's Disease Centre, University College London Institute of Neurology, London, United Kingdom. Tel.: (+31) (0) 71526 2197. Fax: (+31) (0) 715248253. E-mail : N.A.Aziz@lumc.nl

Number of characters in the title: 79

Number of characters in the running head: 41

Number of words in the abstract: 100

Number of words in the manuscript body: 1714

Number of references: 20

Number of tables and figures: 1 table and 2 color figures

Key words: Huntington disease, disease progression, weight loss, body mass index, metabolism, bioenergetics, polyglutamine disorders 


\begin{abstract}
Unintended weight loss is a hallmark of Huntington disease (HD), but it is unknown to what extent weight loss impacts the rate of disease progression. Therefore, using longitudinal data from the EnrollHD study, we assessed the association between baseline body mass index (BMI) and the rate of clinical progression in $5821 \mathrm{HD}$ mutation carriers. We found that high baseline BMI was associated with a significantly slower rate of functional, motor and cognitive deterioration (all $p<0.001$ ), independent of mutant HTT CAG repeat size. Our findings provide strong rationale for exploration of systemic metabolism as a therapeutic target in HD.
\end{abstract}




\section{Introduction}

Huntington disease (HD) is an autosomal dominantly inherited neurodegenerative disorder caused by a CAG repeat expansion in the $H T T$ gene resulting in a long polyglutamine tract in the $\mathrm{N}$-terminus of the encoded protein huntingtin. ${ }^{1}$ Apart from motor, cognitive and behavioral disturbances, a hallmark of the disease is unintended weight loss, both in HD patients and premanifest mutation carriers ${ }^{2,3}$ as well as various genetic animal models of the disease.$^{4-6}$ Previously we found that weight loss in HD patients increases with larger mutant HTT CAG repeat size, ${ }^{2}$ while others reported higher caloric intake in premanifest mutation carriers with increasing CAG repeat length. ${ }^{7}$ These findings suggest a hypermetabolic state in HD which worsens with larger mutant HTT CAG repeat size, possibly due to a polyglutamine length-dependent interference of mutant huntingtin with cellular energy production. ${ }^{4}$ Although in a small study higher body mass index (BMI) was found to be associated with slower disease progression, ${ }^{8}$ subsequent investigations in somewhat larger cohorts could not confirm this finding. ${ }^{9,10}$ Therefore, despite resulting in general weakening and a decline in the quality of life, ${ }^{4}$ it is still unknown to what extent weight loss influences disease progression in HD. Elucidation of the association between body weight and disease progression, however, is of paramount importance as it would not only allow for a better prediction of disease course but could also provide a strong rationale for further exploration of promising energy-based treatments as potential therapeutic targets in HD. ${ }^{11,12}$ In this study, we therefore aimed to provide an accurate and conclusive estimate of the association between body weight and the rate of progression on the most important clinical domains of HD using longitudinal data from the largest cohort currently available.

\section{Materials and Methods}

\section{Observational cohort study}

Participants: For assessing the association between BMI (defined as body weight in kilograms divided by the square of height in meters) and disease progression we used monitored data from 5821 individuals (54\% female) participating in the Enroll-HD study, which also included longitudinal data from 2488 individuals who had previously participated in the Registry study. ${ }^{13,14}$ All participating sites were required to obtain and maintain local ethics committee approvals and all participants gave signed informed consent for their data to be included. ${ }^{13,14}$ We retrieved all data from the Enroll-HD website on February 17, 2017, and included all participants with a mutant CAG repeat size of $\geq 36$ in our analysis. ${ }^{14}$ 


\section{Statistical analyses}

We applied linear mixed-effects models with both fixed and random terms for disease duration to account for the correlation between the repeated measurements on each individual, using an unstructured variance-covariance matrix. ${ }^{15}$ First, we assessed the rate of weight loss by including BMI as the dependent variable and sex, age of onset (defined as the age at clinical diagnosis), disease duration and mutant CAG repeat size as independent variables. Next, we also included an interaction term between disease duration and mutant CAG repeat size to quantify the effect of CAG repeat size on the rate of weight loss. Subsequently, we assessed the effect of baseline BMI - defined as the first BMI measurement recorded for each subject - on measures of disease progression including Unified Huntington Disease Rating Scale (UHDRS) total functional capacity (TFC), total motor score (TMS) and all available UHDRS cognitive sub-scores. In addition, we performed a principal components analysis on the six UHDRS cognitive subdomains to derive a unique summary statistic which could capture most of the variation in the cognitive scores: This analysis resulted in six factors of which the first (principal component 1 (PC1)) accounted for $80.9 \%$ of the variation, while the other factors each accounted for $6.5 \%$ or less of the variation. Therefore, we also used PC1 as a dependent variable. Each measure of disease progression was treated as the dependent variable while disease duration, baseline BMI as well as its interaction with disease duration, sex, age of onset, mutant CAG repeat size and its interaction with disease duration were included as independent variables. For subgroup analyses, we divided the cohort based on baseline BMI in underweight, normal weight, overweight, obese and morbidly obese individuals according to the World Health Organization cut-off points of $<18.5,18.5-<25,25-<30,30-<40$ and $\geq 40 \mathrm{~kg} / \mathrm{m}^{2}$, respectively. To reduce multicollinearity all continuous variables were centered around their respective means. All values are reported as mean \pm standard deviation unless otherwise specified. All tests were two-tailed and the threshold for statistical significance was set at 0.05 . Programming was performed in RStudio Version 1.0.136 (R base 3.3.2) using the 'Ime4' package.

\section{Results}

Weight loss in HD patients

At baseline, BMI ranged from 11.7 to $63.2 \mathrm{~kg} / \mathrm{m}^{2}$ (median: $24.7 \mathrm{~kg} / \mathrm{m}^{2}$, interquartile range: 22.0 to 28.1 $\left.\mathrm{kg} / \mathrm{m}^{2}\right)$. Mean baseline BMI was $25.6 \pm 5.2 \mathrm{~kg} / \mathrm{m}^{2}$ and average BMI declined with 0.06 units $(95 \% \mathrm{Cl}$ : - 
0.08 to $-0.03, \mathrm{p}<0.001$ ) per year. For each extra CAG repeat average BMI was 0.32 units lower $(95 \% \mathrm{Cl}$ : -0.38 to $-0.26, \mathrm{p}<0.001)$ and the rate of weight loss aggravated with $0.02 \mathrm{BMI}$ units/year $(95 \% \mathrm{Cl}:-0.03$ to $-0.01, p<0.001$ ), after adjustment for sex, age of onset and disease duration (Figure 1).

\section{Baseline BMI is a significant predictor of disease progression}

Mean baseline TFC score was $9.9 \pm 3.4$, decreasing by 0.53 points per year $(95 \% \mathrm{Cl}:-0.51$ to -0.55$)$. Each unit increase of baseline BMI was associated with 0.012 points/year slower decline on the TFC scale (95\% Cl: 0.008 to $0.016, p<0.001)$, Figure $2 A$.

The average TMS score at baseline was $24.7 \pm 21.4$, increasing by 3.25 point per year ( $95 \% \mathrm{Cl}: 3.14$ to 3.36). Each unit increase in baseline BMI was related to 0.059 points/year slower rate of deterioration on the TMS scale ( $95 \% \mathrm{Cl}:-0.080$ to $-0.037, \mathrm{p}<0.001)$, Figure 2B.

As expected all UHDRS cognitive sub-scores significantly deteriorated during the follow-up period (Table 1). Higher baseline BMI was associated with a slower rate of decline on all UHDRS cognitive subdomains as well as the cognitive summary score represented by PC1 (Table 1 and Figure 2C).

\section{Sensitivity and subgroup analyses}

Given that our primary analyses were based on the entire dataset which also contained individuals with underweight ( $n=203$ ) or morbid obesity ( $n=96$ ) (Figures 1 and 2 ) we also performed a sensitivity analysis by repeating the calculations after exclusion of these individuals. The results did not change materially and remained statistically significant (data not shown). Similarly, adjustment for disease stage at baseline, as represented by the TFC score, did not change the statistical significance of the results (data not shown). Compared to normal weight participants and after adjustment for sex, age of onset, disease duration and CAG repeat size: 1) In underweight individuals mean TFC score was lower and mean TMS score was higher by 1.12 and 7.82 points, respectively (both $p<0.001$ ), 2) In overweight, obese and morbidly obese individuals mean TMS scores were lower by 2.29, 4.36 and 7.04 points, respectively (all $\mathrm{p}<0.01$ ), and 3 ) the rates of functional and motor deterioration were significantly slower in (morbidly) obese individuals, while the rate of cognitive deterioration was slower in overweight and obese participants (all $p<0.01$ ).

\section{Discussion}


Using high-quality, longitudinal data from the largest cohort of HD mutation carriers available to date, here we present conclusive evidence for an association between body weight and the rate of disease progression in HD. Patients with a high BMI at baseline exhibited a slower rate of progression on all clinical domains which are known to deteriorate with longer disease duration in HD, including functional capacity, motor function and cognitive performance. In addition, we provide more precise estimates of the strong length-dependent detrimental effect of mutant HTT CAG repeat size on body weight and the rate of weight loss, validating and extending our previous findings. ${ }^{2}$ Importantly, however, the association between $\mathrm{BMI}$ and disease progression was independent of mutant CAG repeat size, indicating that factors other than CAG repeat size must account for this relation.

Until now reports regarding a possible link between body weight and disease progression in HD have been discrepant. Although Myers et al. were the first to identify a high BMI as a predictor of slower progression in a group of 42 patients with $\mathrm{HD},{ }^{8}$ it is unknown to what extent baseline differences in mutant HTT CAG repeat size, which is strongly associated with both BMI and disease progression, ${ }^{2}$ might have accounted for their findings. Moreover, subsequent studies found no clear association between body weight and disease progression. ${ }^{9,10}$ However, unequivocal interpretation of these previous reports is further limited by the fact that most of them involved relatively small-scaled studies which were performed before the introduction of the UHDRS, a rating scale which standardized clinical assessment in HD and thereby allowed for a more accurate evaluation of disease progression. ${ }^{16}$ Taking advantage of a much larger cohort of HD mutation carriers who were systematically and uniformly followed up for a considerable amount of time, here we show that a relatively high BMI is indeed a robust and consistent predictor of disease progression in HD. Importantly, the effect of BMI was seen on all clinical measures of progression in HD including functional and motor impairment and decline on all cognitive subdomains of the UHDRS. As the mechanisms governing the rate of motor and cognitive decline in HD are likely to be central, it is tempting to speculate that the association between $\mathrm{BMI}$ and disease progression in HD might be due to an effect of disturbances of energy regulating mechanisms on central nervous system function. ${ }^{4}$ Dysregulation of energy homeostasis in HD could be mediated through pathology of central energy regulating structures, especially the hypothalamus which is known to be affected in $\mathrm{HD},{ }^{17,18}$ or might arise from cellular alterations engendered by mutant huntingtin expression in peripheral tissues. ${ }^{19}$

Our study has certain limitations. First, although our findings indicate that BMI is a strong predictor of disease progression in HD, the observational nature of our study does not permit for direct conclusions 
regarding the directionality or causality of this association. Second, data on body composition were not collected, precluding a further characterization of which body weight components (i.e. fat-free or fat mass) mediate the largest effect. ${ }^{20}$

In conclusion, we found that a high BMI was associated with a slower rate of disease progression in HD independent of mutant HTT CAG repeat size and disease stage. These findings thus suggest that systemic metabolism could influence clinical progression in HD, and should therefore be further explored as a potential target area for therapeutic interventions aiming to modify the rate of progression of HD.

\section{Acknowledgements}

Enroll-HD is a longitudinal observational study for Huntington's disease families intended to accelerate progress towards therapeutics; it is sponsored by CHDI Foundation, a nonprofit biomedical research organization exclusively dedicated to developing therapeutics for HD. Enroll-HD would not be possible without the vital contribution of the research participants and their families. NAA is supported by a VENI-grant (\#91615080) from the Netherlands Organization of Scientific Research and a Marie Sklodowska-Curie Individual Fellowship grant from the European Union (Horizon 2020, \#701130).

\section{Author Contributions}

$J M M v B$ and NAA contributed to the conception and design of the study; JMMvB and NAA contributed to the acquisition and analysis of data; JMMvB, SLG, ACL, GBL, RACR and NAA contributed to drafting the text and preparing the figures.

\section{Conflicts of Interest}

The authors report no conflicts of interest. 


\section{References}

1. The Huntington's Disease Collaborative Research Group. A novel gene containing a trinucleotide repeat that is expanded and unstable on Huntington's disease chromosomes. Cell 1993;72:971-83.

2. Aziz NA, van der Burg JM, Landwehrmeyer GB, et al. Weight loss in Huntington disease increases with higher CAG repeat number. Neurology 2008;71:1506-13.

3. Mochel F, Charles P, Seguin F, et al. Early energy deficit in Huntington disease: identification of a plasma biomarker traceable during disease progression. PLoSONE 2007;2:e647.

4. Aziz NA, Roos RA. Characteristics, pathophysiology and clinical management of weight loss in Huntington's disease. Neurodegener Dis Manag 2013;3:549-59.

5. Skene DJ, Middleton B, Fraser CK, et al. Metabolic profiling of presymptomatic Huntington's disease sheep reveals novel biomarkers. Scientific reports 2017;7:43030.

6. van der Burg JM, Bacos K, Wood NI, et al. Increased metabolism in the R6/2 mouse model of Huntington's disease. NeurobiolDis 2008;29:41-51.

7. Marder K, Zhao H, Eberly S, Tanner CM, Oakes D, Shoulson I. Dietary intake in adults at risk for Huntington disease: analysis of PHAROS research participants. Neurology 2009;73:385-92.

8. Myers RH, Sax DS, Koroshetz WJ, et al. Factors associated with slow progression in Huntington's disease. Arch Neurol 1991;48:800-4.

9. Feigin A, Kieburtz K, Bordwell K, et al. Functional decline in Huntington's disease. Mov Disord 1995;10:211-4.

10. Mahant N, McCusker EA, Byth K, Graham S. Huntington's disease: clinical correlates of disability and progression. Neurology 2003;61:1085-92.

11. Brandhorst S, Choi IY, Wei M, et al. A Periodic Diet that Mimics Fasting Promotes Multi-System Regeneration, Enhanced Cognitive Performance, and Healthspan. Cell Metab 2015;22:86-99.

12. Dupuis L, Pradat PF, Ludolph AC, Loeffler JP. Energy metabolism in amyotrophic lateral sclerosis. The Lancet Neurology 2011;10:75-82.

13. Orth M, Handley OJ, Schwenke C, et al. Observing Huntington's disease: the European Huntington's Disease Network's REGISTRY. Journal of neurology, neurosurgery, and psychiatry 2011;82:1409-12.

14. Landwehrmeyer GB, Fitzer-Attas CJ, Giuliano JD, et al. Data Analytics from Enroll-HD, a Global Clinical Research Platform for Huntington's Disease. Movement Disorders Clinical Practice 2017;4:212-

24.

15. Fitzmaurice GM, Laird NM, Ware JH. Applied longitudinal analysis. Hoboken, New Jersey: John Wiley \& Sons, Inc.; 2004.

16. Huntington_Study_Group. Unified Huntington's Disease Rating Scale: reliability and consistency. Huntington Study Group. Mov Disord 1996;11:136-42.

17. van Wamelen DJ, Aziz NA, Roos RA, Swaab DF. Hypothalamic alterations in Huntington's disease patients: comparison with genetic rodent models. Journal of neuroendocrinology 2014;26:761-75.

18. Hult S, Soylu R, Bjorklund T, et al. Mutant huntingtin causes metabolic imbalance by disruption of hypothalamic neurocircuits. Cell Metab 2011;13:428-39.

19. van der Burg JM, Bjorkqvist M, Brundin P. Beyond the brain: widespread pathology in Huntington's disease. Lancet Neurol 2009;8:765-74.

20. Sussmuth SD, Muller VM, Geitner C, et al. Fat-free mass and its predictors in Huntington's disease. Journal of neurology 2015;262:1533-40. 


\section{Figure legends:}

Figure 1: Weight loss increases with higher CAG repeat size. The graph depicts the relation between $\mathrm{BMI}$ and age with separate regression lines for each category of mutant HTT CAG repeat size. It is evident from this graph that patients with higher CAG repeat size have both a lower body weight and a higher rate of weight loss. The shaded areas around the regression lines indicate the $95 \%$ confidence intervals around the estimates. Median follow-up time was 2.0 years (interquartile range: 0.9 to 3.7 ). Mutant CAG repeat size ranged from 36 to 67 (mean \pm standard deviation: $43.4 \pm 3.5$ ). Inset: Colors represent different categories of CAG repeats based on the indicated cut-off values (note that the first category in red represents carriers of mutations in the reduced-penetrance range).

Figure 2. Higher baseline BMI is associated with slower disease progression in Huntington disease. A higher body mass index (BMI) at baseline was associated with a slower rate of progression on all assessed clinical domains in patients with Huntington disease, including total functional capacity (A), total motor score (B) and a cognitive summary score represented by PC1 (C, please refer to the text for details). All estimates were adjusted for sex, age of onset and mutant CAG repeat size. Each point represents one subject and the shaded areas around the regression lines indicate the $95 \%$ confidence intervals around the estimates. 
Table 1: Effect of baseline body mass index on measures of disease progression in Huntington disease.

\begin{tabular}{|c|c|c|c|}
\hline & Disease duration $^{1}$ & BMI baseline ${ }^{2}$ & Interaction $^{3}$ \\
\hline Total Functional Capacity & $-0.53(-0.55,-0.51)^{* * *}$ & $0.017(-0.002,0.036)$ & $0.012(0.008,0.016)^{* * *}$ \\
\hline Total Motor Score & $3.25(3.14,3.36)^{* * *}$ & $-0.498(-0.606,-0.390)^{* * *}$ & $-0.059(-0.080,-0.037)^{* * *}$ \\
\hline Symbol Digit Modality Test ${ }^{4}$ & $-1.43(-1.49,-1.37)^{* * *}$ & $0.265(0.192,0.338)^{* * *}$ & $0.023(0.011,0.036)^{* * *}$ \\
\hline Stroop Color Naming Test ${ }^{4}$ & $-1.98(-2.08,-1.88)^{* * *}$ & $0.208(0.104,0.312)^{* * *}$ & $0.046(0.026,0.067)^{* * *}$ \\
\hline Stroop Word Reading Test ${ }^{4}$ & $-2.91(-3.05,-2.78)^{* * *}$ & $0.148(0.017,0.280)^{*}$ & $0.058(0.031,0.085)^{* * *}$ \\
\hline Stroop Interference Test ${ }^{4}$ & $-1.09(-1.16,-1.02)^{* * *}$ & $0.093(0.023,0.162)^{* *}$ & $0.029(0.015,0.042)^{* * *}$ \\
\hline Verbal Fluency Test (Categories) $^{4}$ & $-0.53(-0.57,-0.50)^{* * *}$ & $0.067(0.037,0.099)^{* * *}$ & $0.011(0.005,0.018)^{* * *}$ \\
\hline Verbal Fluency Test (Letters) $^{4}$ & $-0.67(-0.75,-0.60)^{* * *}$ & $0.181(0.096,0.266)^{* * *}$ & $0.022(0.007,0.037)^{* *}$ \\
\hline Cognitive Summary Score (PC1) ${ }^{5}$ & $-0.19(-0.20,-0.18)^{* * *}$ & $0.019(0.007,0.030)^{* *}$ & $0.004(0.002,0.006)^{* * *}$ \\
\hline
\end{tabular}

Legend: ${ }^{*} \mathrm{p}<0.05,{ }^{* *} \mathrm{p}<0.01,{ }^{* * *} \mathrm{p}<0.001$

$\left.{ }^{1}\right)$ This column contains the regression coefficients associated with disease duration which can be interpreted as the rate of disease progression per year in units of the particular outcome measure.

$\left.{ }^{2}\right)$ This column contains the regression coefficients associated with baseline BMI which can be interpreted as the average increase or decrease in the outcome measure during the follow-up period per unit increase of baseline BMI.

$\left.{ }^{3}\right)$ This column contains the regression coefficients of the interaction term between baseline $\mathrm{BMI}$ and disease duration: A significant interaction means that baseline BMI affects the rate of disease progression.

$\left.{ }^{4}\right)$ All Unified Huntington Disease Rating Scale cognitive scores indicate the total number of correct items (in 1 minute)

$\left.{ }^{5}\right)$ The first principal component (PC1) of a principal component analysis based on all six Unified Huntington Disease Rating Scale cognitive scores (please refer to the main text for further details). 